\title{
THEORY OF DONOR-BOUND MULTI-EXCITON COMPLEXES IN GERMANIUM
}

\author{
R.S. Pfeiffer \\ Department of Physics, University of California, San Diego, LaJolla, CA 92093, U.S.A.* \\ and \\ Department of Physics, University of Michigan, Ann Arbor, MI 48109, U.S.A. \\ and \\ H.B. Shore \\ Department of Physics, San Diego State University, San Diego, CA 92182, U.S.A.* \\ and \\ Department of Physics, University of Michigan, Ann Arbor, MI 48109, U.S.A.
}

(Recein ed 3 April 1981 by H. Suhl)

\begin{abstract}
We report calculations of ground and excited state energies for multiexciton complexes bound to phosphorus, arsenic, and bismuth donors in germanium. Band structure effects are included in a self consistent density functional calculation. Theoretical recombination spectra derived from our results agree very well with existing data. The results imply the existence of a kinetic bottleneck to the growth of large complexes.
\end{abstract}

ELECTRONS AND HOLES have long been known to form bound pairs, called excitons, and there has been much interest in the question of larger aggregates of electrons and holes, especially since the discovery of bound excitons, of bound multi-exciton complexes (BMEC), and of large electron-hole droplets. In this paper we deal with BMEC and their recombination spectra for several donor elements in germanium. It is now generally accepted that each line in a BMEC spectrum is the result of the recombination of an electron and a hole from a system formed from a neutral donor and $m$ excitons, called a BMEC of size $m$. The qualitative features of the spectra can be accounted for by a "shell model" [1], where it is assumed that the excitons dissociate when the complex forms and the now separate electrons and holes go into specific single particle orbitals.

There have been relatively few observations of BMEC lines in germanium [2-4], but the well studied series of lines found in silicon are well accounted for by this model $[5,6]$. Each line corresponds to the decay of a complex from size $m$ to $m-1$, with the configurations (i.e. the assignment of electrons and holes to shell model orbitals) of the initial and final states differing only in the removal of one electron and one hole.

In order to calculate the recombination spectra of BMEC in silicon and germanium correctly, it is

*Permanent address. necessary to include such band-structure effects as the splitting of donor $s$ states, central-cell corrections to the potential of the donor atom, and coupling between the light and heavy hole bands. The early densityfunctional calculations of BMEC energies used simple models which omitted these effects $[7,8]$. We have recently calculated energies of BMEC states in silicon which include band-structure effects, and have obtained results which agree reasonably well with experimental data [9]. Silicon is, however, a bad candidate for theoretical study due to the extreme cubic anisotropy of the light and heavy hole bands, and the close proximity of the split off $J=1 / 2$ hole band.

Germanium, with its more nearly spherical valence band edge can be expected to produce theoretical energies in better agreement with observed spectra than was the case for silicon, and thus provides a better test of the applicability of the density functional approach. Indeed, our results for germanium, which we report here, are in excellent agreement with experiment. The results can also be used to predict the expected locations of those series of lines which have not been observed in germanium though their counterparts are well known in silicon.

We use the density-functional theory of Hohenberg and Kohn $[10,11]$ to obtain self-consistent differential equations for Hartree-like electron and hole wavefunctions, and solve these equations numerically. This allows us to use methods devised for calculating 
donor and acceptor energies to obtain electron and hole energies respectively.

The external potential of our problem, which represents the electrostatic potential of a bare donor, includes an empirical central-cell correction. The potential has the following form:

$$
V(r)=\left(e / \epsilon_{0} r\right)\left[1+Z^{\prime} \exp \left(-r / r_{0}\right)\right] \text {. }
$$

We adjust the parameters $Z^{\prime}$ and $r_{0}$ for each impurity type to fit the experimental donor ground state and valley-orbit splitting. Once $Z^{\prime}$ and $r_{0}$ are set, there are no other adjustable parameters.

To obtain the valley orbit splitting of the donor $1 \mathrm{~s}$ energies, as well as the electron energies for larger complexes, we use the method of Resca and Resta [12]. The splitting derives from the fact that the conduction band of germanium has four equivalent minima. If we assume that the electron wavefunctions have $k$-components near these minima, we can construct a single particle wavefunction of the form:

$\psi^{\Gamma}(r)=\sum_{j=1}^{4} \alpha_{j}^{\Gamma} \phi\left(\mathbf{K}_{j}, \mathbf{r}\right) F_{j}^{\Gamma}(r)$

Here $\phi\left(\mathbf{K}_{j}, \mathbf{r}\right)$ is the Bloch wave of an electron at the $j$ th minimum $\mathbf{K}_{j}$. The $\alpha_{j}$ are coefficients which give $\psi^{\Gamma}$ an overall symmetry of $\Gamma_{1}$ or $\Gamma_{5}$. For example, a wavefunction of symmetry $\Gamma_{1}$ has all $\alpha_{j}=1 / 2$.

We assume the $F_{j}^{\Gamma}(r)$ envelope functions are the same for all $j$ for a particular wavefunction and that in the case of $1 s$ orbitals, which are the only ones needed for $m \leqslant 8$, they are spherically symmetric. To solve for $F^{\Gamma}(r)$, the potential $V(r)$ in the effective mass Hamiltonian is replaced by $\widetilde{V}^{\Gamma}(r) \equiv V(r) G^{\Gamma}(r)$, where $G^{\Gamma}(r)$ is the spherical average of $\left|\Sigma \alpha_{j} \phi\left(\mathbf{K}_{j}, \mathbf{r}\right)\right|^{2}$.

We note that the above method is completely valid only if the overlap of envelope functions between different valleys is negligible. For the shallow electron levels considered here, the functions are well localized at the bottoms of the valleys; the effects of overlap slightly modify $Z^{\prime}$ and $r_{0}$ but do not significantly alter the calculated energies.

To obtain hole energies and wavefunctions, we employ the spherical approximation of Baldereschi and Lipari [13]. Their model Hamiltonian treats holes as spin-3/2 particles and couples orbitals of angular momentum $L$ and $L+2 . F$ (where $\mathbf{F}=\mathbf{L}+\mathbf{J}$ ) and $F_{z}$ are good quantum numbers of the Hamiltonian. Thus: the ground state is a mixture of $s$ and $d$ orbitals with no nodes and $F=3 / 2$ which, following the notation of [13], we denote $1 s_{3 / 2}$. The lowest excited hole orbital is a mixture of $p$ and $f$ orbitals, also with no nodes and $F=3 / 2$, which we denote $2 p_{3 / 2}$. The two states have cubic group symmetries of $\Gamma_{8}^{+}$and $\Gamma_{8}^{-}$respectively. Our numerical procedure differs from that of [13], since that work employs parametrized variational wave functions, while the requirement of self-consistency forces us to solve the $2 \times 2$ coupled differential equations numerically, using a procedure based on the Runge - Kutta method [14].

Table 1

\begin{tabular}{|c|c|c|c|c|}
\hline \multirow[t]{2}{*}{$m$} & \multirow{2}{*}{$\begin{array}{l}\text { Configuration } \\
\text { electrons; holes }\end{array}$} & \multicolumn{3}{|c|}{$|E| \mathrm{meV}$} \\
\hline & & $\mathbf{P}$ & As & $\mathrm{Bi}$ \\
\hline \multirow[t]{2}{*}{0} & $\Gamma_{1}$ & 12.74 & 14.12 & 12.58 \\
\hline & $\Gamma_{5}$ & 10.03 & 10.04 & 9.82 \\
\hline \multirow[t]{4}{*}{1} & $2 \Gamma_{1} ; \Gamma_{8}^{+}$ & 18.43 & 20.15 & 18.22 \\
\hline & $2 \Gamma_{1} ; \Gamma_{8}^{-}$ & 17.77 & 19.40 & 17.60 \\
\hline & $\Gamma_{1}, \Gamma_{5} ; \Gamma_{8}^{+}$ & 16.92 & 18.01 & 16.69 \\
\hline & $\Gamma_{1}, \Gamma_{5} ; \Gamma_{8}^{-}$ & 16.40 & 17.48 & 16.18 \\
\hline \multirow[t]{4}{*}{2} & $2 \Gamma_{1}, \Gamma_{5} ; 2 \Gamma_{8}^{+}$ & 23.10 & 24.66 & 22.86 \\
\hline & $2 \Gamma_{1}, \Gamma_{5} ; \Gamma_{8}^{+}, \Gamma_{8}^{-}$ & 22.34 & 23.82 & 22.12 \\
\hline & $\Gamma_{1}, 2 \Gamma_{5} ; 2 \Gamma_{8}^{+}$ & 21.83 & 22.80 & 21.59 \\
\hline & $\Gamma_{1}, 2 \Gamma_{5} ; \Gamma_{8}^{+}, \Gamma_{8}^{-}$ & 21.16 & 22.14 & 20.91 \\
\hline \multirow[t]{4}{*}{3} & $2 \Gamma_{1}, 2 \Gamma_{5} ; 3 \Gamma_{8}^{+}$ & 28.62 & 30.02 & 28.32 \\
\hline & $2 \Gamma_{1}, 2 \Gamma_{5} ; 2 \Gamma_{8}^{+}, \Gamma_{8}^{-}$ & 27.78 & 29.19 & 27.52 \\
\hline & $\Gamma_{1}, 2 \Gamma_{5} ; 3 \Gamma_{8}^{+}$ & 27.48 & 28.37 & 27.19 \\
\hline & $\Gamma_{1}, 3 \Gamma_{5} ; 2 \Gamma_{8}^{+}, \Gamma_{8}^{-}$ & 26.71 & 27.58 & 26.43 \\
\hline \multirow[t]{4}{*}{4} & $2 \Gamma_{1}, 3 \Gamma_{5} ; 4 \Gamma_{8}^{+}$ & 34.77 & 36.08 & 34.42 \\
\hline & $2 \Gamma_{1}, 3 \Gamma_{5} ; 3 \Gamma_{8}^{+}, \Gamma_{8}^{-}$ & 33.88 & 35.19 & 33.58 \\
\hline & $\Gamma_{1}, 4 \Gamma_{5} ; 4 \Gamma_{8}^{+}$ & 33.75 & 34.54 & 33.40 \\
\hline & $\Gamma_{1}, 4 \Gamma_{5} ; 3 \Gamma_{8}^{+}, \Gamma_{8}^{-}$ & 32.89 & 33.69 & 32.60 \\
\hline \multirow[t]{4}{*}{5} & $2 \Gamma_{1}, 4 \Gamma_{5} ; 4 \Gamma_{8}^{+}, \Gamma_{8}^{-}$ & 40.51 & 41.73 & 40.18 \\
\hline & $2 \Gamma_{1}, 4 \Gamma_{5} ; 3 \Gamma_{8}^{+}, 2 \Gamma_{8}^{-}$ & 39.61 & 40.81 & 39.28 \\
\hline & $\Gamma_{1}, 5 \Gamma_{5} ; 4 \Gamma_{8}^{+}, \Gamma_{8}^{-}$ & 39.60 & 40.33 & 39.25 \\
\hline & $\Gamma_{1}, 5 \Gamma_{5} ; 3 \Gamma_{8}^{+}, 2 \Gamma_{8}^{-}$ & 38.72 & 39.45 & 38.40 \\
\hline 6 & $2 \Gamma_{1}, 5 \Gamma_{5} ; 4 \Gamma_{8}^{+}, 2 \Gamma_{8}^{-}$ & 46.67 & 47.79 & 46.32 \\
\hline
\end{tabular}

In Table 1, we list the calculated energies of the ground and several excited configurations for complexes of sizes up to $m=5$, and the ground state energy for $m=6$. We list only configurations in which each electron or hole is in its ground or lowest excited orbital. The values in Table 1 represent the energies of the listed configurations with respect to an equal number of well separated electrons and holes. We have investigated configurations other than those reported here to make sure that there are no other states of lower energy than those listed.

The various series of BMEC lines are defined by specifying the initial configuration and the recombination of a particular electron and hole; this uniquely determines the final configuration. Thus an $\alpha_{m}$ line has a BMEC of size $m$ in its ground state as its 
initial configuration and involves the recombination of a $\Gamma_{1}$ electron and a $\Gamma_{8}^{+}$hole. A $\beta_{m}$ line starts with a BMEC of size $m+1$ in its ground state and involves the recombination of a $\Gamma_{5}$ electron and a $\Gamma_{8}^{+}$hole. A $\gamma_{m}$ line has an initial complex of size $m+1$, with one electron thermally promoted from a $\Gamma_{1}$ to a $\Gamma_{5}$ orbital, and involves the recombination of a $\Gamma_{1}$ electron and a $\Gamma_{8}^{+}$ hole. There are also several observed single lines, defined as follows $[3,4]$.

$$
\begin{aligned}
\delta: \quad m=1\left(\Gamma_{1} \Gamma_{5} ; \Gamma_{8}^{+}\right) \rightarrow \Gamma_{1} \text { donor, } \\
\epsilon: \quad m=1\left(2 \Gamma_{1} ; \Gamma_{8}^{-}\right) \rightarrow \Gamma_{1} \text { donor, } \\
\alpha_{1} \Gamma_{5}: \quad m=1\left(2 \Gamma_{1} ; \Gamma_{8}^{+}\right) \rightarrow \Gamma_{5} \text { donor. }
\end{aligned}
$$

The " $\alpha_{1} \Gamma_{5}$ " line represents a violation of the shellmodel, since the transition requires the promotion of an electron from $\Gamma_{1}$ to $\Gamma_{5}$ in addition to the recombination of the $\Gamma_{1}-\Gamma_{8}^{+}$pair.

The predicted photon energy of a no-phonon replica is obtained from Table 1 by evaluating $E_{G}-\left|E_{m+1}\right|+\left|E_{m}\right|$, where we use $E_{G}=744.64 \mathrm{meV}$ for the gap energy. The BMEC lines which have so far been found by Mayer and Lightowlers [3, 4] in germanium are $\alpha_{1}, \alpha_{2}, \gamma_{1}, \delta, \epsilon$, and $\alpha_{1} \Gamma_{5}$; we compare these experimental lines for phosphorus donors with our theoretical values in Fig. 1. As Fig. 1 indicates, the calculated energies are within $0.5 \mathrm{meV}$ of the experimental values.

The most striking difference between experiment and theory is that the experimental $\gamma_{1}, \alpha_{2}$ and $\delta$ lines are split into multiplets. This is probably due to term splittings within the $m=1\left(\Gamma_{1} \Gamma_{5} ; \Gamma_{8}^{+}\right)$configuration [3]. One might expect that the non-spherical interaction between electrons and holes would split this configuration into four sublevels; i.e. $\Gamma_{5} \times \Gamma_{8}=\Gamma_{6}+\Gamma_{7}+2 \Gamma_{8}$. This would entail four $\alpha_{2}, \gamma_{1}$ and $\delta$ lines each, consistent with the interpretation of Mayer and Lightowlers $[3,4]$. Larger complexes could produce more elaborate term splittings. It remains unclear why these splittings are so much more pronounced in $\mathrm{Ge}$ than in $\mathrm{Si}$.

Table 1 can be used to predict the positions of lines not yet observed in $\mathrm{Ge}$, including the $\alpha$ series for larger complexes and the important $\beta$ series, which results from ground state to ground state transitions. These lines are predicted to be rather closely spaced as compared to their Si counterparts, and may be obscured by the $\gamma, \delta, \epsilon$ lines. However, another possibility arises from the result that the $m=2$ BMEC in its ground state is bound by only $E_{B}=0.49 \mathrm{meV}$ with respect to dissociation into an $m=1 \mathrm{BE}$ and a FE. An experimental value for $E_{B}$, estimated from the data of [3], is about $0.80 \mathrm{meV}$. At an experimental temperature of $4 \mathrm{~K}$, the ratio $E_{B} / k_{B} T$ is therefore only about $1.5-2.3$. With such a small ratio, and with reasonable background densities

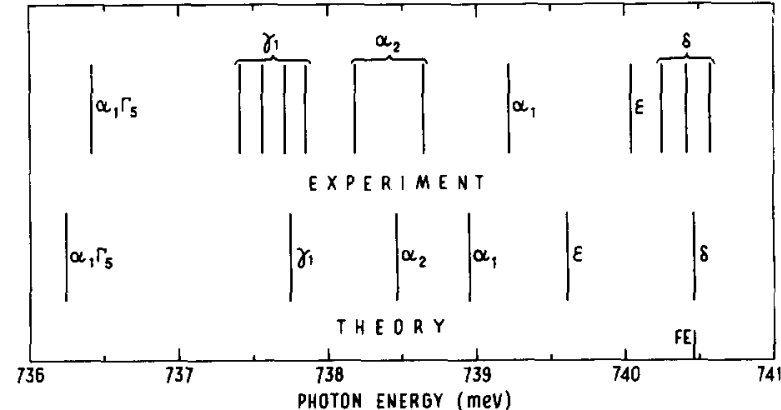

Fig. 1. Comparison of experimental and theoretical recombination spectra for phosphorus impurities in $\mathrm{Ge}$. The free exciton line (FE) is shown for comparison.

of free excitons, the population of $m \geqslant 2$ complexes would be extremely small. At low enough temperatures it may be possible to overcome this bottleneck.

The results of this study provide strong support for the shell model, but also raises an interesting question concerning the interpretation of the single particle orbitals. In Hartree-Fock, the BMEC are not bound with respect to decay into free excitons [15]. Thus unlike the situation in ordinary atoms, correlation energy plays an essential role in holding this system together. Therefore the use of single particle orbitals cannot mean that the true wave function is described by a single Slater determinant. The density functional method, while providing a powerful computational tool, obscures the difficulty by simply assigning the same exchange-correlation energy density as an infinite uniform electron-hole liquid of the same density [16-18]. There seems no doubt as to the utility of the density functional method; for example in the model problem of a single BE without band structure effects the density functional calculation of Wünsche and Henneberger [18] gives essentially identical results to the variational calculation of Stébé and Munschy [19], which involves a fully parametrized three-particle wavefunction. In summary, we regard the great success of the shell model in predicting allowed and forbidden transitions between BMEC states as requiring deeper explanation than has so far been given, perhaps involving a quasiparticle interpretation of the shell model orbitals.

Acknowledgement - This work was completed while both of us were visitors at the University of Michigan. We wish to thank the U.M. Physics Department for their hospitality. The work was supported by NSF grant DMR 79-20813.

\section{REFERENCES}

1. G. Kirczenow, Solid State Commun. 21, 713 (1977); Can. J. Phys. 55, 1787 (1977).

2. R.W.Martin, Solid State Commun. 14, 369 (1974). 
3. A.E. Mayer \& E.C. Lightowlers, J. Phys. C12, L539 (1979); J. Phys. C12, L945 (1979).

4. A.E. Mayer \& E.C. Lightowlers, J. Phys. C13, L747 (1980).

5. See for example, M.L.W. Thewalt, Proc. 14th Int. Conf. Phys. Semicond., p. 605. Edinburgh (1978) (and references therein).

6. M.L.W. Thewalt, Can J. Phys. 55, 1463 (1977).

7. H.-J. Wünsche, K. Henneberger \& V.E. Khartsiev, Phys. Status Solidi (b) 86, 505 (1978).

8. H.B. Shore \& R.S. Pfeiffer, Proc. 14th Int. Conf. Phys. Semicond., p. 627. Edinburgh (1978).

9. H.B. Shore \& R.S. Pfeiffer, Proc. 15th Int. Conf. Phys. Semicond., Kyoto (1980) p. 453.

10. P. Hohenberg \& W. Kohn, Phys. Rev. 136, B864 (1964).

11. W. Kohn \& L.J. Sham, Phys. Rev. 140, A1133 (1965).
12. L. Resca \& R. Resta, Solid State Commun. 29, 279 (1979).

13. A. Baldereschi \& N.O. Lipari, Phys. Rev. B8, 2697 (1973).

14. K.S. Mendelson \& H.M. James, J. Phys. Chem. Solids 25, 729 (1964).

15. Y.C. Chang \& T.C. McGill, Phys. Rev. Lett. 45, 471 (1980).

16. Refs. [7], [8], and [9] discuss the role of correlation energy in BMEC density functional calculations in some detail.

17. H.-J. Wïnsche, Phys. Status Solidi (b) 96, 379 (1979).

18. H.-J. Wünsche \& K. Henneberger, Phys. Status Solidi (b) 91, 331 (1979).

19. B. Stébé \& G. Munschy, Solid State Commun. 35, 557 (1980). 